\title{
An Adaptive Proportional Resonant Controller for Single Phase PV Grid Connected Inverter Based on Band-Pass Filter Technique
}

\author{
H. Khalfalla, Student IEEE Member, S. Ethni, Maher Al-Greer, IEEE Member, V. Pickert, IEEE Member, M. \\ Armstrong, Van Thang Phan \\ School of Electrical and Electronic Engineering, Newcastle University, Newcastle upon Tyne, United Kingdom \\ hamza.khalfalla@ncl.ac.uk, salaheddine.ethni@ncl.ac.uk, maher.al-greer@ncl.ac.uk, volker.pickert@ncl.ac.uk, \\ matthew.armstrong@ncl.ac.uk, vantung.phan@ncl.ac.uk
}

\begin{abstract}
This paper presents an adaptive proportional resonant (PR) controller for single phase grid connected inverter that adapts its control parameters to grid impedance variations. Forth order band bass filter is designed and then integrated with the adaptive scheme for on-line detection of any variations in the resonance frequency. The estimated frequency is then processed by statistical signal processing operation to identify the variations in the grid impedance. For the on-line tuning of the PR parameters, a look-up table technique is utilized and its parameters are linked with the estimated impedance values. Simulation results based on MATLAB environment clearly verify the effectiveness of the proposed control scheme for $2 \mathrm{~kW}$ grid connected inverter system.
\end{abstract}

Keywords-Adaptive Proportional Resonant Controller; Grid Impedance Estimation; LCL Filter, Look-up Table.

\section{INTRODUCTION}

Renewable energy sources like wind turbines (WT) and photovoltaic cells (PV) are clean and affordable. Special attention has been given to the PV grid connected inverters and it is expected to experience large growth in the future due to cost reduction and increasing efficiencies [1]. However, the interaction between the PV inverter system and the distribution grid produces power quality (PQ) implications such as, harmonic generation, DC injection, islanding, and voltage level variation. Consequently, certain stringent standard requirements and limitations such as IEEE929 and IEEE1547 have been established and must be considered for designing such systems [2, 3]. These standards are satisfied by means of robust control schemes where the output current of the inverter system is regulated and synchronized to the grid voltage. Therefore, many control algorithms have been presented in the literature including predictive controller, hysteresis controller, linear proportional integral (PI) and proportional resonant (PR) controllers [4]. Typically, the PI controller is widely used because of its simplicity and ease of implementation; however, in power grid applications, the PI controller has some drawbacks such as inability to track reference sinusoidal signals in single phase systems without a steady state error and poor capabilities of rejecting disturbances due to the dynamic of the integral action. These drawbacks can be alleviated by using the PR controller as a main control loop. A PR controller is able to track sinusoidal reference signals and reject the disturbance within the system in high performance. At the fundamental frequency, the PR controller produces an infinite gain; therefore, the steady state error can be eliminated [5]. Several techniques of resonant controllers have been presented in the literature [6-9]. In order to comply with power quality standards the voltage source inverter (VSI) is interfaced to the utility grid via a low pass filters to filter out the high harmonic components produced by the high switching VSI frequency. Among all, the LCL filter is preferable because it can provide superior filtering action compared to other types. However, the LCL filter is a third order filter which adds more challenges to the design of the control loop [10]. Two damping methods (passive and active) can be utilized to eliminate the resonance peak of LCL filter [11, 12] and both techniques ensure the stability and improve the overall system performance. Noticeably, the fixed control loop is incapable to compensate for the aforementioned issues and for the variations of the PV system condition such as changes in the grid impedance and grid frequency; these variations obviously affect the performance of the system. From this prospective more emphasis has been given to advanced control methods such as adaptive PR controller (APR) for grid connected inverter system. In [13], an adaptive control scheme was proposed in which the sampling and switching frequencies were adapted to the grid frequency. A frequency APR controller was considered in $[14,15]$. An approach to adapt PR controller to the time varying grid impedance in a state space control has been proposed in [11]. The authors in [16] presented a grid impedance adaptation method based on a feed forward control of the grid voltage. In this paper a new APR for single phase grid connected PV inverter is proposed where the grid impedance variation is detected and estimated and with this knowledge the control loop is tuned for high performance operation. The paper is organized as follows: Section II describes the simulation of the system and its interaction with 
the utility grid. In Section III the proposed APR controller and the design of bandpass filter with signal processing are presented. Simulation results of the close loop system have been given in Section IV. Finally, conclusions are drawn in the last section.

\section{SYSTEM SIMULATION AND DESCRIPTION}

Fig. 1 shows the block diagram of the proposed adaptive PR control structure. It is worth to mention that this work is concerning about the grid side control with an assumption of fixed input voltage from the PV source. Here, the grid impedance $\left(L_{g}\right)$ is connected in series with the LCL filter; therefore, any change in $L_{g}$ will change the filter resonant frequency as given in (1).

$$
w_{\text {res }}=\sqrt{\frac{L_{1}+L_{2}+L_{g}}{L_{1}\left(L_{2}+L_{g}\right) C_{f}}}
$$

Where, $w_{\text {res }}$ is the resonance frequency, $L_{1}$ is the inverter side inductor, $L_{2}$ represents grid side inductor, $C_{f}$ is filter capacitor and $L_{\mathrm{g}}$ is the grid impedance. The changeable resonant frequency can be used as an indication for any variation of grid impedance. From this, equation (1) can be rearranged to calculate the grid impedance as:

$$
L_{g}=\frac{L_{1}-w_{r e s}{ }^{2} L_{1} L_{2} c_{f}}{w_{r e s}{ }^{2} L_{1} c_{f}}
$$

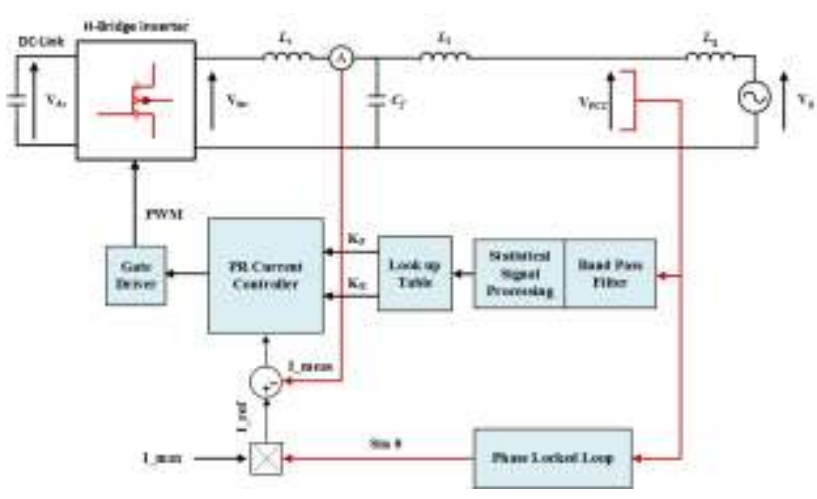

Fig. 1. Block diagram of the proposed adaptive PR controller.

From (1), the resonant frequency is significantly affected by the grid impedance variation. As demonstrated in Fig. 2, when the grid impedance increases the resonant frequency and the controller bandwidth decrease which may deviate the system towards instability.

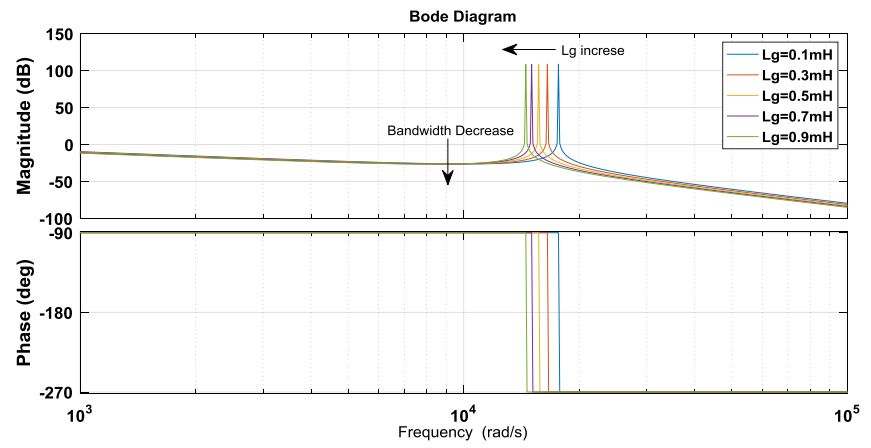

Fig. 2. Bode plot diagram of non-damped LCL filter under wide range of grid impedance variation

The closed loop control system in Fig.1 can be simplified as depicted in Fig.3.

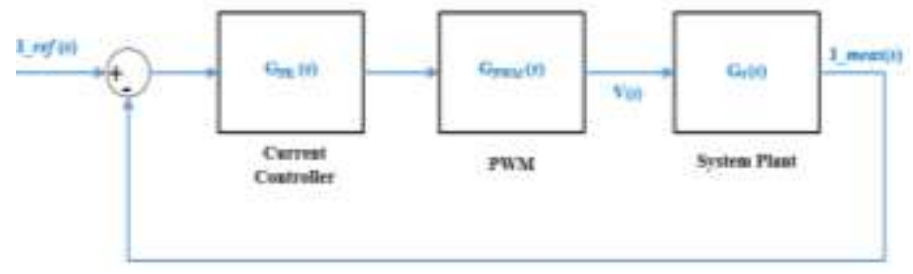

Fig. 3. Block diagram of the current control system

The control loop gains are expressed as:

$$
\text { Loop Gain }=G_{P R}(s) G_{P W M}(s) G_{f}(s)
$$

Where, $\mathrm{G}_{P R}(s)$ is the PR control model, $\mathrm{G}_{P W M}(s)$ is the pulse width modulation model, and $\mathrm{G}_{f}(s)$ is the plant model including the damping resistance. In the case of the inverter side current sensing all three transfer functions become[17]:

$$
\begin{gathered}
\mathrm{G}_{P R}(s)=k_{p}+\frac{2 w_{c} k_{i} s}{s^{2}+2 w_{c} s+w_{0}^{2}} \\
\mathrm{G}_{P W M}(s)=\frac{1}{1+1.5 T_{S} s} \\
\mathrm{G}_{f}(s)=\frac{1}{L s} \frac{\left(s^{2}+\mathrm{R}_{\mathrm{d}} Z_{L C}^{2} s+Z_{L C}^{2}\right)}{\left(s^{2}+\mathrm{R}_{\mathrm{d}} \mathrm{C}_{f} w_{r e s}^{2} s+w_{r e s}^{2}\right)}
\end{gathered}
$$

Where, $T_{s}$ is the sampling period of the current controller, $R_{d}$ filter damping resistance which connected in series to the filter capacitor, $\mathrm{Z}^{2}{ }_{L C}=\left[L_{g T} C_{f}\right]^{-1}$, and $L_{\mathrm{gT}}=L_{\mathrm{g}}+L_{2}$

Now, a fourth-order band-pass filter block is inserted into the control loop to monitor to filter out the voltage signal at the point of common coupling (VPCC) (Fig. 1). The filter is designed to filter only the harmonic components around the resonant frequency $\left(w_{\text {res }}\right)$ which cause the oscillation in the voltage signal. The output signal of the band-pass filter has been processed in such a way that it can be readable by the controller 
for parameters tuning. This process can be achieved by statistic signal processing techniques consisting of signal rectification, integration and mean value determination. It is important to mention that the output current of the inverter (Fig.1), is synchronized with the grid voltage by the use of phase locked loop (PLL) to operate the system as close as possible to unity power factor [15].

\section{THE PROPOSED ADAPTIVE PR CONTROLLER (APR)}

\section{A. Proportional Resonance (PR) Controller.}

Ideally, the transfer function of PR controller is described as:

$$
G_{P R}(s)=k_{p}+\frac{2 k_{r} s}{s^{2}+w_{0}^{2}}
$$

Where, $K_{p}$ and $K_{r}$ are the proportional and resonance gains respectively. Comparable to PI controller, $\mathrm{K}_{\mathrm{r}}$ is responsible for eliminating the steady state error while $K_{p}$ is tuned such that good transient response and stability are guaranteed [6]. In practical application, the infinite gain associated with the ideal case might lead to instability problems. Alternatively; nonideal PR controller as given in [16], is applied to improve the performance of the controlled system:

$$
G_{P R}(s)=k_{p}+\frac{2 w_{c} k_{r} s}{s^{2}+2 w_{c} s+w_{0}^{2}}
$$

In $(8), \omega_{\mathrm{c}}$ is the cutoff frequency which is introduced to add more flexibility for selecting the bandwidth of the controller and to reduce the sensitivity towards the variation of background grid frequency [17]. Fig.4 shows the comparison between the frequency responses of an ideal and non- ideal PR controller. At the fundamental frequency, a high gain is achieved to eliminate the steady state error whereas approximately no gains appear at other frequencies.

Another valuable advantageous of PR controller is the possibility of cascading several selective harmonic compensators $(\mathrm{HC})$ to the controller (9) for further reduction of current total harmonic distortion $\left(3^{\text {th }}, 5^{\text {th }}\right.$ and $\left.7^{\text {th }}\right)$. The resonant controller parameters in this case are tuned at the frequencies of interest to be compensated for [18].

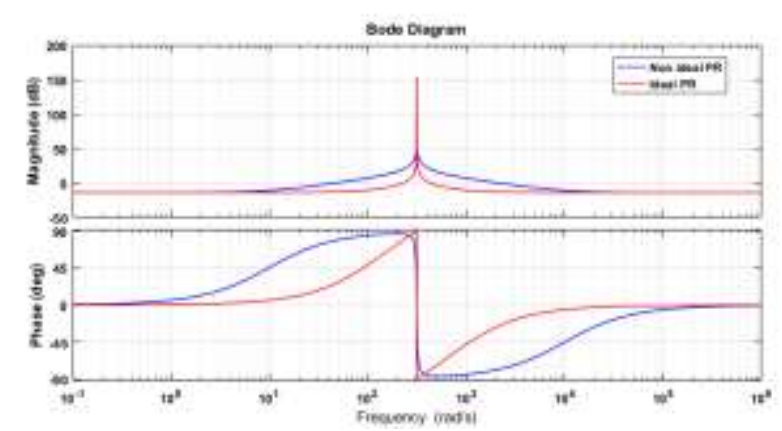

Fig. 4 Bode plot diagram of an ideal and non-ideal PR controller.

$$
P R(s)_{h}=\sum_{h=3,5,7}^{n} k_{p}+\frac{2 w_{c} k_{r} s}{s^{2}+2 w_{c} h s+\left(h w_{0}\right)^{2}}
$$

Where, $h$ is the order of harmonics.

\section{B. Band Pass Filter Desgin for Resonant Detection}

Bandpass filter are widely used in communication and signal processing application for frequency selection at pre specified range. In this work, a fourth order $\left(4^{\text {th }}\right)$ bandpass filter with high corner cut off frequency attenuation rate is designed for resonance detection purposes. The targeted harmonic components at the resonance frequency are relatively small in magnitude compare to fundamental components. For this reason, Sallen-Key topology with Butterworth response is proposed to achieve high order response and to minimize the ripple at the filter pass band respectively. Analogue filter was selected to ensure that the digital current control loop is not compromised with the long duration of the required computation time needed for resonant detection. In addition to its simplicity, the main valuable advantage of such topology is the capability of allowing high quality factor combined with high pass gain without the use of inductors. The passive components and design process of the filter were achieved according to [19], in which the design process of bandpass filter has been extensively discussed. The transfer function of second order bandpass filter is given in (10), and the two cascaded second order bandpass that is yielded a desired 4th order filter is depicted in Fig. 5.

$$
H(s)=\frac{\left(1+\frac{R_{b}}{R_{a}} s\right) \frac{s}{R_{a} C_{1}}}{s^{2}+\left(\frac{1}{R_{1} C_{1}}+\frac{1}{R_{2} C_{1}}+\frac{1}{R_{2} C_{2}}-\frac{R_{b}}{R_{a} R_{f} C_{1}}\right) s+\frac{R_{1} R_{f}}{R_{1} R_{2} R_{f} C_{1} C_{2}} s}
$$

The transfer function of desired $4^{\text {th }}$ order filter is provided by multiplication of two $2^{\text {th }}$ order bandpass filter transfer functions which yields to equation (11).

$$
H_{B P}(s)=H_{1}(s) H_{2}(s)
$$

Where, $H_{1}(s)$ and $H_{2}(s)$ are the transfer function of first and second stages of $2^{\text {th }}$ order filter respectively.

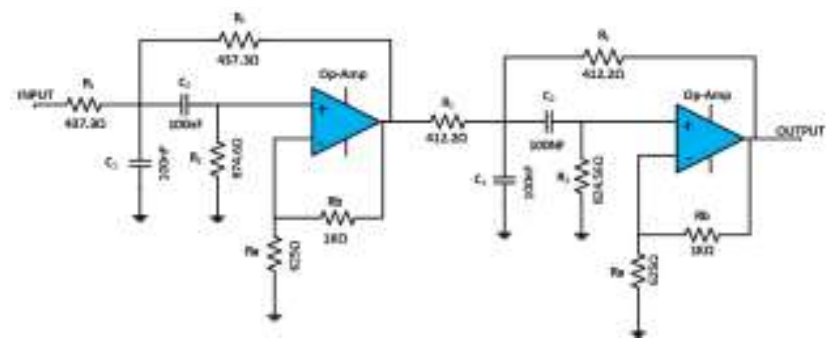

Fig. 5. Circuit diagram of 2 cascaded bandpass filter.

As presented in the Fig. 6, the band pass gain is significantly magnified for ease of resonant detection and centered at 3,750 $\mathrm{Hz}$ with a bandwidth of $1,500 \mathrm{~Hz}$. This centered frequency is 
determined in accordance to the resulted resonance frequency from the equation given earlier in (1). The bandwidth of the bandpass filter is chosen to cover all the expected resonance frequency deviations caused by the variation of $L_{g}$.

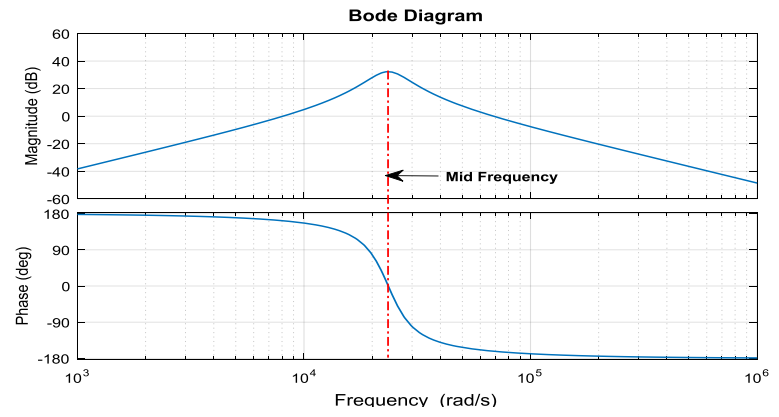

Fig. 6. Frequency response of the $4^{\text {th }}$ order bandpass filter.

\section{Statistic Signal Processing TeChNiques for ONLINE ADAPTATION OF CONTROLLER PARAMETERS}

The filtered voltage waveform at the output of the bandpass filter is followed by a statistic signal processing techniques that rectify and integrate the signal. Eventually, the mean value of the integrated signal is taken. As the grid impedance changes the mean value will also change yielding into estimating any variation in the grid impedance. Finally, the estimated grid impedance value is used as a pointer to the look up table for appropriately selecting the corresponding PR parameters $\left(K_{p} \&\right.$ $K_{r}$ ) (Fig. 7). A two-dimensional look up table is built and its values are selected based on trial and error method. However, optimization techniques can be employed for optimally tuning the PR parameters. This, however, might add more computation time and complexity to the system.

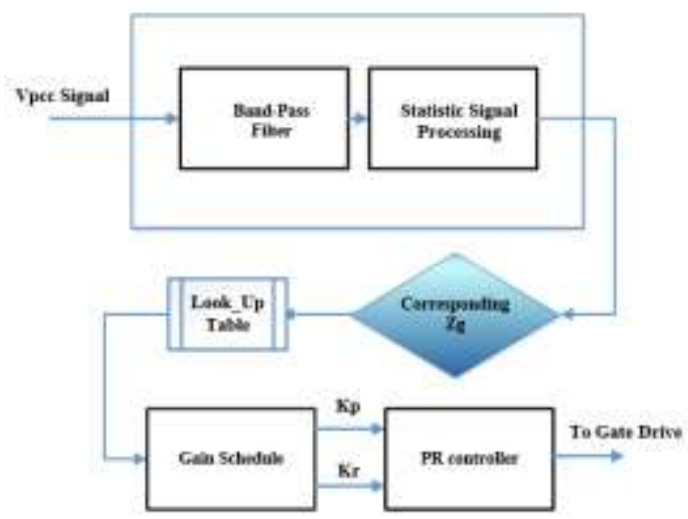

Fig. 7. Gains schedule of APR controller.

\section{SIMULATION RESULTS}

To evaluate the concept of the proposed APR controller, a single phase grid system shown in Fig. 1 has been simulated using MATLAB Simulink. Note that the grid voltage has been simulated with different magnitudes and phase angles of up to the $40^{\text {th }}$ harmonic orders; all harmonics are summed together representing a real case emulation (see Fig. 8).

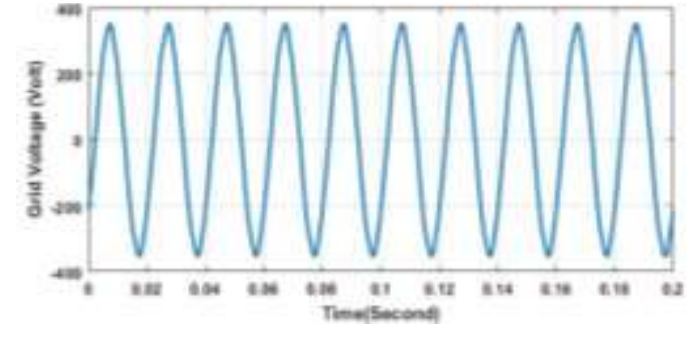

Fig. 8. Simulation result of emulated grid voltage.

The converter specification and parameters are given in Table I and Table II respectively. According to IEC60725 standard [20], for a single phase distribution supply in UK, the maximum grid impedance is $0.4 \Omega$ and $0.789 \mathrm{mH}$. Thus, the proposed adaptive PR controller has been tested under wide range of grid impedance variation starting from $0.1 \mathrm{mH}$ for a stiff grid up to $0.8 \mathrm{mH}$ for a weak grid.

TABLE I

CONVERTER SPECIFICATION

\begin{tabular}{ccc}
\hline Symbol & Parameter & Value \\
\hline $\mathbf{P}_{\mathbf{o}}$ & Output Power & $2 \mathrm{KW}$ \\
$\mathbf{V}_{\mathbf{d c}}$ & DC-Link Voltage & $400 \mathrm{VDC}$ \\
$\mathbf{V}_{\mathbf{g}}$ & Grid Voltage & $240 \mathrm{VAC}$ \\
$\mathbf{f}_{\mathbf{s w}}$ & Switching Frequency & $10 \mathrm{kHz}$ \\
\hline \multicolumn{3}{c}{ TABLE II } \\
\hline $\mathbf{S y m b o l}$ & CONVERTER PARAMETERS \\
\hline $\mathbf{L}_{\mathbf{1}}$ & Parameter & Value \\
$\mathbf{L}_{\mathbf{2}}$ & Inverter Side Inductor & $2.12 \mathrm{mH}$ \\
$\mathbf{C}_{\mathbf{f}}$ & Grid Side Inductor & $0.45 \mathrm{mH}$ \\
$\mathbf{f}_{\text {res }}$ & Filter Capacitor & $3.53 \mu \mathrm{F}$ \\
\hline
\end{tabular}

The obtained simulation results are compared to conventional PR controller which parameters are fixed. Results (Table.III) clearly shows that the proposed APR scheme offer superior harmonic characteristic performance compare to the conventional PR controller.

TABLE III

COMPARISON RESULTS OF CONVENTIONAL AND APR CONTROLLER

\begin{tabular}{|c|c|c|c|c|}
\hline \multirow{2}{*}{$\begin{array}{c}L_{g} \\
(\mathrm{mH})\end{array}$} & \multicolumn{2}{|c|}{ APR } & \multicolumn{2}{|c|}{ PR } \\
\hline & $\begin{array}{c}\text { Ig_THD } \\
\%\end{array}$ & $\underset{\%}{\text { Vpcc_THD }}$ & $\begin{array}{c}\text { Ig_THD } \\
\%\end{array}$ & $\underset{\%}{\text { Vpcc_THD }}$ \\
\hline 0.2 & 0.55 & 2.47 & 0.99 & 2.50 \\
\hline 0.4 & 0.54 & 2.48 & 0.99 & 2.55 \\
\hline 0.6 & 0.61 & 2.49 & 0.94 & 2.59 \\
\hline 0.8 & 0.57 & 2.49 & 0.95 & 2.63 \\
\hline
\end{tabular}

Fast Fourier Transform analysis (FFT) has been carried out for the inverter output current under the adaptive and conventional PR controller. As illustrated in Fig. 9a and Fig. 9b the frequency spectrum of the grid current under APR control is less than that of conventional PR control. 


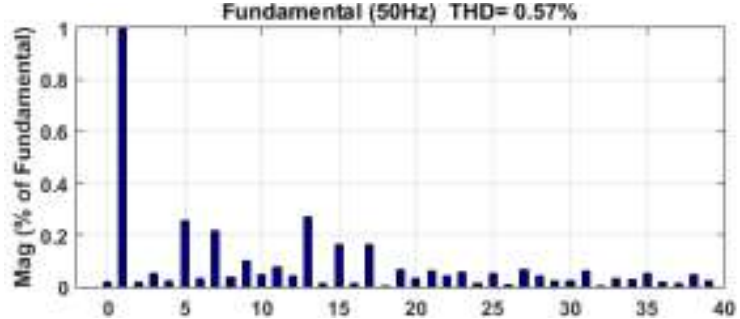

(a)

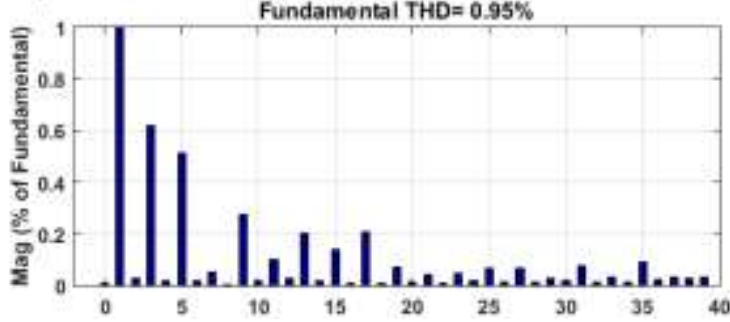

(b)

Fig.9. FFT analysis of grid current. (a) APR controller. (b).PR controller

Fig. 10 shows the THD of the grid current over time for a system where the grid impedance abruptly varies from $0.2,0.4$, 0.6 , and $0.8 \mathrm{mH}$ in a $0.2 \mathrm{~s}$ interval each. As presented in Fig. 10, the PR parameters are tuned in accordance to grid impedance variation. The PR control parameters $\left(K_{p}\right.$ and $\left.K r\right)$ are changing to keep the THD of the grid current at acceptable low levels (according to the IEEE929 limit of 5\%). In each case, the proposed adaptive controller shows very promising results and can handle any variations in the grid impedance. It is worth to mention that the observed high THD during switching is only an effect of the abrupt change of the inductor value during the simulation. Finally, simulation results of a harmonic polluted grid voltage with injected grid current under the proposed control strategy is shown in Fig. 11. Here, the injected grid current is synchronized and regulated under a wide range of grid impedance variation. This demonstrates the effectiveness and robustness of the proposed APR.
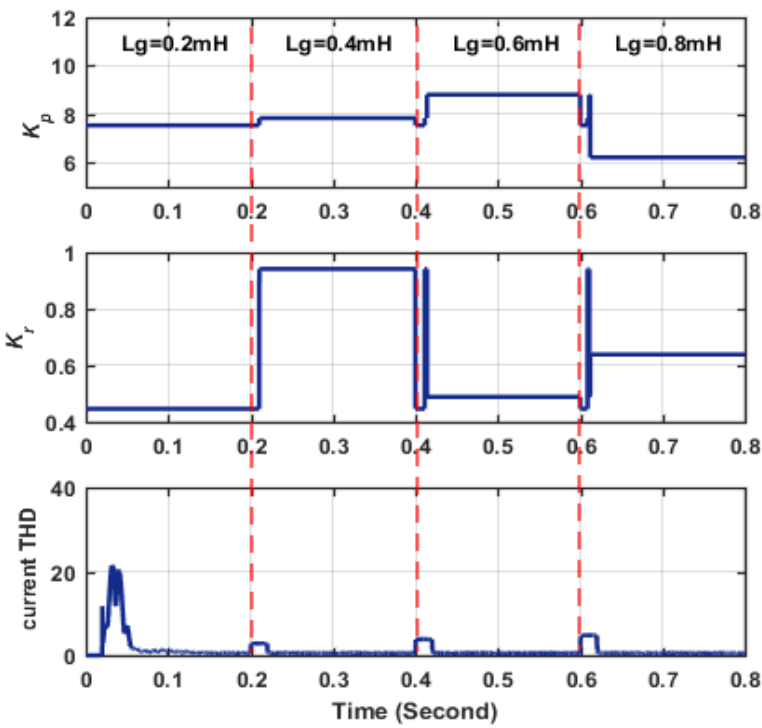

Fig. 10. Online adaptation of the APR control parameters.

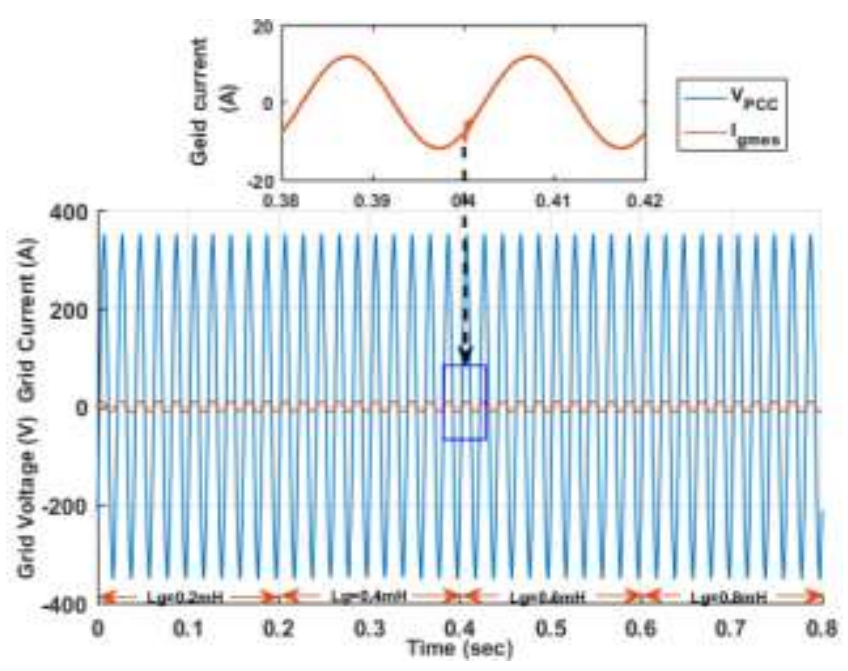

Fig. 11. Grid voltage and current waveforms under changeable grid impedance with the proposed control strategy.

\section{CONCLUSION}

A new control strategy based on an adaptive proportional resonant (APR) controller has been developed and successfully tested on a simulated $2 \mathrm{~kW}$ single phase grid tide PV inverter. A fourth order Sallen-Key bandpass filter tailored to the system to capture the harmonic components around the resonant frequency has been implemented. Statistic signal processing technique was employed in order to provide the controller with signals corresponded to the changeable grid impedance. A considerable low level of current total harmonic distortion (THD) is achieved in comparison with conventional PR controller and compliance with IEEE929-Standard has been demonstrated.

\section{REFERENCES}

[1] S. Kouro, J. I. Leon, D. Vinnikov, and L. G. Franquelo, "Grid-Connected Photovoltaic Systems: An Overview of Recent Research and Emerging PV Converter Technology," IEEE Industrial Electronics Magazine, vol. 9, pp. 47-61, 2015.

[2] "IEEE Recommended Practice for Utility Interface of Photovoltaic (PV) Systems," in IEEE Std 929-2000, ed, 2000.

[3] "IEEE Draft Application Guide for IEEE Standard 1547, Interconnecting Distributed Resources With Electric Power Systems," in IEEE Unapproved Draft Std P1547.2/D11, Sept 2008, ed, 2008, p. 1.

[4] H. M. El-Deeb, A. Elserougi, A. S. Abdel-Khalik, S. Ahmed, and A. M. Massoud, "An adaptive PR controller for inverter-based distribution generation with active damped LCL filter," in 2013 7th IEEE GCC Conference and Exhibition (GCC), 2013, pp. 462-467.

[5] W. L. Chen and J. S. Lin, "One-Dimensional Optimization for Proportional-Resonant Controller Design Against the Change in Source Impedance and Solar Irradiation in PV Systems," IEEE Transactions 
on Industrial Electronics, vol. 61, pp. 1845-1854, 2014.

[6] T. Ye, N. Dai, C. S. Lam, M. C. Wong, and J. M. Guerrero, "Analysis, Design, and Implementation of a Quasi-Proportional-Resonant Controller for a Multifunctional Capacitive-Coupling Grid-Connected Inverter," IEEE Transactions on Industry Applications, vol. 52, pp. 4269-4280, 2016.

[7] P. Yao, C. Hu, and Y. Lu, "Photovoltaic gridconnected inverter based on frequency feedback PR controller," in 2016 IEEE 11th Conference on Industrial Electronics and Applications (ICIEA), 2016, pp. 2558-2560.

[8] A. Lidozzi, M. D. Benedetto, S. Bifaretti, L. Solero, and F. Crescimbini, "Resonant Controllers With Three Degrees of Freedom for AC Power Electronic Converters," IEEE Transactions on Industry Applications, vol. 51, pp. 4595-4604, 2015.

[9] R. E. Carballo, F. Botterón, G. G. Oggier, and G. O. García, "Design approach of discrete-time resonant controllers for uninterruptible power supply applications through frequency response analysis," IET Power Electronics, vol. 9, pp. 2871-2879, 2016.

[10] S. Eren, M. Pahlevaninezhad, A. Bakhshai, and P. K. Jain, "Composite Nonlinear Feedback Control and Stability Analysis of a Grid-Connected Voltage Source Inverter With LCL Filter," IEEE Transactions on Industrial Electronics, vol. 60, pp. 5059-5074, 2013.

[11] J. R. Massing, M. Stefanello, H. A. Grundling, and H. Pinheiro, "Adaptive Current Control for GridConnected Converters With LCL Filter," IEEE Transactions on Industrial Electronics, vol. 59, pp. 4681-4693, 2012.

[12] C. Bao, X. Ruan, X. Wang, W. Li, D. Pan, and K. Weng, "Step-by-Step Controller Design for LCL-Type Grid-Connected Inverter with Capacitor-CurrentFeedback Active-Damping," IEEE Transactions on Power Electronics, vol. 29, pp. 1239-1253, 2014.

[13] M. Abusara, S. Sharkh, and P. Zanchetta, "Adaptive repetitive control with feedforward scheme for gridconnected inverters," IET Power Electronics, vol. 8, pp. 1403-1410, 2015.

[14] F. Gonzalez-Espin, G. Garcera, I. Patrao, and E. Figueres, "An Adaptive Control System for ThreePhase Photovoltaic Inverters Working in a Polluted and Variable Frequency Electric Grid," IEEE Transactions on Power Electronics, vol. 27, pp. 42484261, 2012.

[15] Y. Yang, K. Zhou, and F. Blaabjerg, "Enhancing the Frequency Adaptability of Periodic Current Controllers With a Fixed Sampling Rate for GridConnected Power Converters," IEEE Transactions on Power Electronics, vol. 31, pp. 7273-7285, 2016.

[16] M. Cespedes and J. Sun, "Adaptive Control of GridConnected Inverters Based on Online Grid Impedance
Measurements," IEEE Transactions on Sustainable Energy, vol. 5, pp. 516-523, 2014.

[17] M. Liserre, F. Blaabjerg, and R. Teodorescu, "Grid Impedance Estimation via Excitation of LCL -Filter Resonance," IEEE Transactions on Industry Applications, vol. 43, pp. 1401-1407, 2007.

[18] A. Javadi, A. Hamadi, A. Ndtoungou, and K. AlHaddad, "Power Quality Enhancement of Smart Households Using a Multilevel-THSeAF With a PR Controller," IEEE Transactions on Smart Grid, vol. 8, pp. 465-474, 2017.

[19] Z. M. A. Zin Ma Ma Myo, Zaw Min Naing, "Design and Implementation of Active Band-Pass Filter for Low Frequency RFID (Radio Frequency Identification) System," in Proceedings of the International MultiConference of Engineers and Computer Scientists, 2009.

[20] H. S. Goh, M. Armstrong, and B. Zahawi, "The effect of grid operating conditions on the current controller performance of grid connected photovoltaic inverters," in 2009 13th European Conference on Power Electronics and Applications, 2009, pp. 1-8. 